7th International Workshop on Astronomy and

Relativistic Astrophysics (IWARA 2016)

International Journal of Modern Physics: Conference Series

Vol. 45 (2017) 1760076 (8 pages)

(C) The Author(s)

DOI: $10.1142 / \mathrm{S} 201019451760076 \mathrm{X}$

\title{
Concluding Remarks to the IWARA 2016
}

\author{
Jorge E. Horvath \\ Departamento de Astronomia \\ Instituto de Astronomia Geofísica e Ciências Atmosféricas (IAG) \\ Universidade de São Paulo (USP) \\ R. do Matão, 1226, Butantã, 05508-090 São Paulo, SP, Brazil \\ foton@iag.usp.br
}

Published 15 August 2017

Keywords: Particle Physics, Astrophysics, Gravitation, Cosmology.

\section{Introduction}

This is the 7th edition of the IWARA Workshop, this time held in Gramado, RS, Brazil with an excellent attendance and variety of subjects. By its very character, the IWARA Workshop is very open to new ideas and attempts to bring together the experimental results and theoretical/phenomenological work. As stated in the former editions, it is a very difficult task to summarize such an activity, and because of that I apologize in advance to the colleagues that may be misunderstood or misquoted. However, it is the consensus of the Organizing Committee that a Concluding Remarks talk is useful for many participants, specially the younger ones, and serves as a benchmark and a corollary of the event. Since an ad-hoc committee revised and discussed the poster contributions, I will not address them here. What we learned from the participants is summarized below.

\section{The Sessions}

The opening session featured Thomas Böller describing the ambitious survey 4 MOST, expected to cover at least $3 \times 10^{7}$ sources, many of them also registered by GAIA and eROSITA. It is very likely that such an effort could reveal unexpected features, something that happens every time we look deeper. A cherished goal, namely to peep around the event horizon of selected AGNs is still far from the required accuracy of $\mu$ arcsec, but work is in progress.

This is an Open Access article published by World Scientific Publishing Company. It is distributed under the terms of the Creative Commons Attribution 4.0 (CC-BY) License. Further distribution of this work is permitted, provided the original work is properly cited. 


\section{J. E. Horvath}

J. Albert lectured on a series of results obtained by the BaBar experiment focusing the $B^{0}$ decays. The target was the measurements of the $C P$ and $T$ asymmetries, which are believed as sacred building blocks of modern particle physics. Presently the collaboration features around 600 physicists, whereas other groups (i.e. LIGO) are over 1000. It is a sign of the times that such a large volume of people and resources must be spent to study these subtle issues, and one may wonder if the mood of doing physics (al least a large part of it) has been changed forever from the mostly individualistic early 20th century benchmarks.

On a completely different mood, H. Takai presented a lecture which may be subtitled "small is (still) beautiful". He described a desktop, inexpensive experiment to monitor a large number of muon showers. Remarkably, the experiment performed well to detect a very interesting phenomenon, namely the thermal atmospheric tides. The initial disbelief in the student's results lead him to state a powerful no-go theorem ("students are never right") that awaits a full demonstration.

J. Albert returned to describe his beautiful baby ALTAIR, an experiment now devoted to measure and reduce the photometric errors of high-z supernovae. As is well-known, this is a crucial issue to make progress towards the actual cosmological model, with a constant value of dark energy or evolving quantities as a prime feature under discussion.

A timely presentation by A. Lazzarini discussed in detail the breakthrough of gravitational wave detection now widely recognized. He presented the state-of-theart "banana" regions to localize GW150914, which are still large but will offer independent assessment of the location of the sources with increasing accuracy in the future. No NS-NS merging has been seen yet (in spite that these systems were the expected ones for the first detections). It is worthwhile to remark that we are already learning a lot about astrophysics and gravitation itself. As an example, P. Hess discussion of pseudo-complex gravity showed that within his framework the GW signal should correspond to $\sim 1000 M_{\odot}$ black holes, of the so-called intermediate mass regime. This observation highlights the importance of theory-experiment feedback in its full dimension.

Structure formation, and the activity of AGNs were addressed by J. Pacheco. With the help of extensive simulations performed using the NICE numerical code he showed that toroidal structures of molecular $H_{2}$ form frequently around central black holes. In addition, he found that flaring is frequent (but not for $\operatorname{Sgr} A^{*}$ ). It is interesting that large GW rates from this phenomenon are predicted for LISA.

T. Storchi-Bergmann also lectured on AGNs but approaching the observational side. She tried to understand the influx of matter, which is regulated in turn by ejection. This type of feedback is one of the mechanisms considered to determine the stellar Initial Mass Function, and one might wonder whether an "AGN IMF" can emerge from it, with a hint of explanation of the correlation between the velocity dispersion and central BH mass.

The presentation of $\mathrm{R}$. Rosa addressed the nature of large-scale correlations (formerly modeled with fractal math...), which in his view may be emerging from 


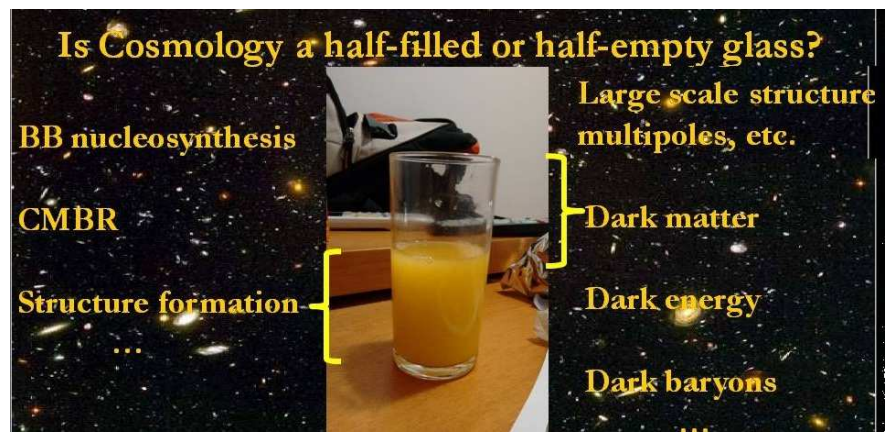

Fig. 1. Cosmology today. Left: most resonant successes. Right: big fundamental question marks.

anomalous diffusion. This issue can be connected with Wen Zhao's talk, who showed that large-scale anomalies are still presenting the latest Planck data. Along a similar line of thought, L. Santos's talk was devoted to polarization studies probing several scales. Actually, polarization was the main issue behind the results of BICEP2, now attributed to contamination.

An overall reflection on present Cosmology is in order here: although it is undeniable that we know much more about the large-scale structure and its evolution, the number of very fundamental questions has not decreased. Can we state whether Cosmology is a half-empty or a half-filled glass (Fig. 1)? There are fierce advocates of both sides within us, and it must be stated that both have very good reasons to argue.

Moving away from cosmological issues, a series of talks addressed neutron stars rotation and related stuff. F. Weber discussed in detail the possible change of topology of a neutron star subject to increasing angular velocities, showing that there may be a transition from a "plain donut" to a "berliner" (Fig. 2). It remains to be seen if ultra-fast pulsars do behave like that, and under which equations of state.

The issue of deformed neutron stars (not necessarily by rotation) was tackled by O. Zubairi from a general point of view. Even in its axisymmetric version, this is a tricky 2D GR problem. An important goal is to tie the deformation to its physical causes, a point sometimes overlooked by GR people but a crucial issue for the real world.

In addition, the crust of the neutron star may be heavily influenced by rotation, as discussed by S. Schramm. For example, its thickness and equilibrium nuclei. Since most timing and transport phenomena depend on the crust, it is important to clarify the nature of the inner crust-outer crust region for a variety of situations.

A related problem, namely the thermo-rotational evolution of a cooling/braking neutron star was discussed by R. Negreiros, with particular interest in the induction of ("continuous") phase transition by the braking process. This is a long standing problem in Astrophysics and I would suggest to tackle it from an observational 

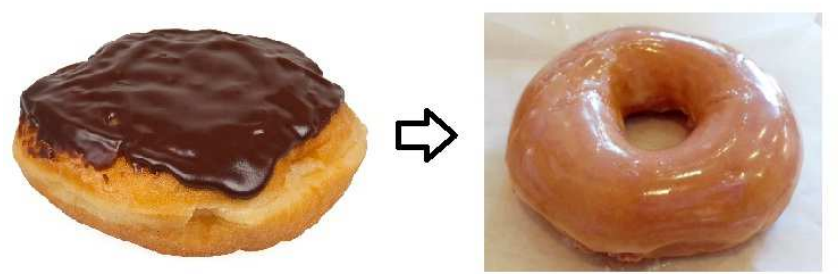

Fig. 2. Extremely rotating neutron stars. A flattened structure (left) may turn into a hollow one (right) provided the equation of state falls in the right range. See Weber's talk for details.

point of view as well, specially now that large facilities (i.e. SKA) are coming up and allow monitoring a large number of sources in real time.

A concrete and exciting system (Cas A) displaying an interesting behavior was discussed by G. Contreras. He remarked that postulating a phase transition to quarks driven by a high-mass star forces the event to have taken place very recently. The source in Cas A is being monitored and reanalyzed by several independent groups to establish definitively the need of a change in the cooling curve.

Work on the bulk structure of neutron stars has been presented within different views. Some examples are R. Farias' presentation describing the exploration of asymmetric chiral matter at finite density, reporting a reconciliation with lattice results. The existence of a critical point indicated by lattice results is still on the works in this issue. A related work was presented by N. Scoccola, focusing on the dependence of the critical temperature for deconfinement $T_{c}$ with the magnetic field $B$. The issue has two parts: first is wether $T_{c}$ grows with $B$ or otherwise, and second if this is relevant for actual physical systems. The non-local approach has a spatial scale of $\sim(1 \mathrm{GeV})^{-1}$ and ultimately resembles the Schwinger-Dyson ladder.

R. Gomes and collaborators took a more traditional approach to construct an extended version of the equation of state for neutron (hyperon) matter. The old controversy about the sudden softening of the equation of state when the hyperon Fermi seas are open reveals a lot of complexity, in particular, the one related to threebody forces which may be "guilty" of massive neutron stars by adding substantial repulsion. In addition, the question of the importance of magnetization beging to be explored to assess realistic situations.

L. Paulucci lectured on an issue which became very timely since the detection of a late infrared excess in two short gamma-ray bursts ("kilonovae"). She considered what happens if the underlying model is not the merging of two NS, but rather two strange stars made of stable strange matter. The ejected exotic matter gives rise to a normal $n-p$ matter with a huge neutron fraction, and therefore the nucleosynthesis proceeds in a "dense Big Bang" fashion, instead of producing lanthanides as in conventional models. It is clear that a lot of systematics remain to be explored to link this proposal to real events, although the observed ones can be reproduced in full. 
R. Valentim revisited the neutrino emission of SN 1987A, showing that within a Bayesian approach, a double peak in time (likely associated with a late phenomenon such as a phase transition to quarks) is preferred over a single continuous light curve. Several models of this late emission are under consideration and, of course, calculations that will be useful for the next supernova.

M.G.B. de Avellar presented his work on the modelling of QPOs, and the possibility of extracting valuable information of the central compact star from them. The physical identification of QPOs as Keplerian frequencies of matter located at the innermost stable orbit is still not confirmed, and becomes important to link a physical cause for the QPOs with the light curves that might be generated by synthetic models.

The next session started with the presentation by S. Burdin, who discussed Higgs physics and the experiments performed at ATLAS-DM experiment. DM particles if produced may be identified by missing transverse moment accounting. On the other hand, the nature of the Higgs particle (elementary or composed?) is still unclear, and the expected SUSY did not show up yet. This situation leads to question whether there is "new physics" before the GUT scale, and one of the possible windows could be CR showers seen by Auger which are produced at center-of-mass values well above the LHC. Given that no money or new acceleration techniques are needed, it may be a worthy effort.

M.B. Gay gave a convincing glimpse of the huge amount of information still hidden in the hadronic spectrum. In particular, she concentrated in perturbative calculations (i.e. quarkonium) for which the data provides a variety of tests. Later on, C. Mariotto discussed small $x$ physics with minimal assumptions, in an attempt to address the so-called "pocket $\sigma_{e f f}$ " beyond known parametrizations, a feasible but painful task indeed.

A loosely related talk given by J. Nogueira addressed the three-body decays of $B^{+} \mathrm{s}$ with minimal hypothesis as a tool to study possible $C P$ violations. It must be stressed that $C P T$ is a very cherished building block of our physical world, one that the philosopher I. Lakatos would call a "hard core" of almost all physical theories. This is why the search for anomalies becomes so interesting and lively. With a similar spirit J. Solano explored the issue of $D$ meson decays. As it stands, the landscape of them is a big mess, but important surprises can remain hidden into it and justifies the hard work.

One remarkable claim backed up by a set of calculations was made by A. Garat, namely that in spite of the insistence on the separation between internal and spacetime symmetries, they could be mixed and thus blurred in the Einstein-Maxwell system. Without invoking any super-symmetry, this feature is extremely important since it may point towards a quantization of gravity along known schemes. This is certainly a topic to be followed up.

An interesting review of higher dimensional theories, presented by O. Piguet, brought also fundamental issues to the discussion. In particular, the construction of compactified theories with the de Sitter or anti-de Sitter symmetry group as a 
5-D basis brings in the possibility of having a fundamental length (which could be great for cosmology), but complicates the identification of spinors and other issues. It must be kept in mind that the de Sitter group can not be continuously deformed into the Poincaré group and this is why a great deal of caution must be taken when constructing such theories.

Gravitation was the subject of a group of talks, for instance, P. Moraes focused on the interesting question of compact star structure in $f(R, T)$ theories, showing that some particular versions make the maximum mass to grow beyond the corresponding TOV value, although it is not clear by how much.

J. O'Brien addressed conformal gravity present in string theory, in particular he discussed the properties of the element $(1-2 M / r+\gamma r)$, which amusingly appears very similar to a vacuum solution of Rastall gravity discussed by Oliveira, Velten and Fabris (2016). Connections between seemingly unrelated theories are always welcome by gravitation colleagues.

J. Pereira gave a stimulating talk in which he discussed how the convert the forthcoming Satellite Test of Equivalence Principle into a test of the MOND (perhaps TeVs?) theory, gauging its relevance properly. In a similar fashion, H. Velten dedicated his talk to show that the velocity of gravitation can not differ more than $1 \%$ from electromagnetism using the Hulse-Taylor pulsar. Both talks serve to show how to question fundamental "truths" towards a deeper understanding of gravity features.

P. Chardonnet lectured on his models having a pair-instability SN as GRB engine. He discussed how oxygen burning in a fully turbulent regime can produce observed variable ligth-curves. Since GRBs rate deviates from the SMF at high redshifts, this feature may favor massive, low-metallicity progenitors as required. This talk was complementary to the one by J. Rangel Lemos classifying the lightcurves and attempting to make sense of the variety of cases. It must be remembered that in the early days of BATSE results great efforts were devoted to seek "the" model of GRB, a task that now seems very naive indeed.

Turning to the instrumentation/experiments, C. Frajuca described the Brazilian efforts to operate the resonant mass antenna NIOBE intended to contribute to the GW search and detection. It is clear that in spite of the potential, some important issues are still a concern, for example, the non-gaussian noise produced bu CR deposition at these very high sensitivities.

On the other hand, J. Braga lectured on a satellite project which may boost highenergy research in Astrophysics, namely the missing MIRAX, a wide field coded mask $\mathrm{X}$ ray detector working around $\sim 100 \mathrm{keV}$, an important energy window of processes around neutron stars and black holes. The project seems healthy and will benefit from the participation of former members of the Rossi Timing Explorer.

Two additional talks highlighted the bright prospects for Brazil as a world-class actor in the science game. U. Barres de Almeida presented the Brazilian Science Data Center supported by the generous work of a growing community. This kind 
of facilities may be thought as the modern equivalent of the Alexandria Library of the Ancient World.

The status and prospects of the Cerenkov Telescope Array (CTA) was reviewed by R. Nemenn. An extensive list of science goals is set for this ambitious and timely experiment, well within the 21st century.

M. Alves discussion of the cosmological GW background reinforced the idea that this is one of the most difficult relicts to measure, and it is likely to be over-hidden by foreground binaries.

The construction of a mathematically sophisticated and physically oriented Nbody code was the subject of G. Ferrari's talk. It is known that a lot of crosschecking is needed to validate mutually this kind of efforts to simulate the hierarchy of structure formation.

M. Malheiro devoted his talk to describe models of very massive WD, well above the Chandrasekhar limit allowed not by some change in the behavior of the magnetized equation of state, but rather by the action of Lorentz forces provided the magnetic field reaches $\sim 10^{13} G$. It seems that super-Chandrasekhar objects, the simplest explanation for extremely brilliant SNIa requiring a large amount of synthesized nickel could exist with masses up to $\sim 2 M_{\odot}$ or so, their direct search (for instance, in the SDSS survey) would be in order.

The contraponto of the latter work was the presentation by S.O. Kepler in which he discussed the tentative identification of very-low mass WD formed in binary evolution. There is surely some contamination of ordinary red dwarf in this sample, but the issue is important because it helps refining the stellar evolution models for these quite abundant systems.

The issue of UEHCR was very well represented in the IWARA 2016. Two talks described the results of the Auger Observatory, R. Almeida on the search for largescale anisotropies (which are found to be small, with the obvious exception of the dipole) and the expectation that the "metagalaxy" (halo and beyond in the old Russian denomination) should dominate above $\sim 1 \mathrm{EeV}$. A small unexplained excess is seen at $\sim 22^{\circ}$. Later E. Silva addressed the composition of the primaries, in which a heavier component sets in around $18.5 \mathrm{EeV}$, possibly due to ordinary light nuclei. An important feature is that the number of muon showers initiated by nucleons deviates substantially from the predicted, perhaps indicating a failure of hadronic models at these extreme energies.

The latter was precisely the topic of E. Luna's talk, attempting to derive total cross-sections in a QCD-based approach for center-of-mass energies $\sqrt{s} \sim 10^{5}$ corresponding to typical Auger values. Needless to say, this task is quite a long-shot challenge.

R. Lima challenged the nature of SGR/AXP sources showing that most of them could be ordinary pulsars, but only if the internal structure is described by a nonTOV set of equations championed by the ICRA group. Alternatively, the whole set of objects may be explained within WD models. 


\section{J. E. Horvath}

N. Magalhães addressed the persistent question of non-canonical (i.e. $<3$ ) braking indexes in observed pulsars. The inclusion of extra forces/torques can reconcile the observations and theory, although the physical causes for the former and their viability remain to be studied.

P. Ferreira lectured on the topic of entropy associated to (apparent) horizons. He argued that something is seriously changing during a bounce of a dynamical system. Dark fluids inside a dynamical universe were shown to be problematic, for which an asymptotically de Sitter universe will also develop an event horizon sometime.

R. Sultanov revisited the problem of cooling of molecular gases, a topic of great interest for structure formation. He showed that the cooling time has been overestimated up to now, therefore galaxies would start to form quite earlier. A full integration of these results into codes awaits its turn.

H. Pérez Rojas calculations of a self-magnetization of quantum systems lead him to identify large-scale jets as produced by this phenomenon. Even if a lot of macroscopic effects could be competitive, it would be fascinating if $k p c$-scale objects could be initiated by quantum physics. Time will tell.

I take the opportunity to highlight the very open attitude of all participants to contribute scientifically to the success of this meeting and the whole past series. I believe that the initial intention of setting a forum for the Brazilian High-energy people with connections to Particle, Cosmology and many other related topics has been achieved due to the effort of the Organizers (with César Vasconcellos as its head) and the participants alike.

\section{Acknowledgments}

J.E. Horvath acknowledges research grants from CNPq Federal Agency and FAPESP (Process No 2013/26258-4) and the excellent hospitality of the gauchos at Gramado. 